\title{
New Thermodynamics: Reversibility, Entropy and Adiabatic Processes
}

\author{
Kent W. Mayhew
}

\section{ABSTRACT}

\begin{abstract}
Accepted assertions concerning entropy and reversibility in both isentropic and adiabatic processes will be investigated and challenged. The ultimate conclusion being that accepted thermodynamics is an over-complication.

Furthermore, the need for natural logarithmic functions will be explained. This functionality no longer depends upon entropy, whatever entropy's true guise turns out to be. Moreover, it will be discussed how isothermal entropy change is embedded in circular logic.
\end{abstract}

Keywords: entropy, reversibility, adiabatic, isentropic, circular logic
Published Online: April 27, 2020

ISSN: $2684-4451$

DOI : 10.24018/ejphysics / 2020.2.2.7

K.W. Mayhew*

Independent Researcher not institutionalized (e-mail: kent.mayhew@gmail.com)

*Corresponding Author

\section{INTRODUCTION}

Entropy $(S)$ is a term that was coined by Rudolf Clausius. Strangely, few can agree upon its true meaning after nearly 170 years of use. This author has previously asserted that entropy is nothing short of a mathematical contrivance [1].

Adiabatic is derived from the Greek word adiabatikos, which means that "heat is not able to go through" [2]. Adiabatic processes imply that the systems are either $100 \%$ thermally insulated, or that the processes are too rapid for thermal energy to be exchanged. In either case, the result is that in an adiabatic process, a system does not exchange energy with its surroundings.

Traditional theory starts with a first law equation for an expanding gas, such as:

$$
d Q=d E+P d V
$$

where $d Q$ is the energy input, $d E$ is the change to the system's internal energy, and $P d V$ is the work done in an expanding system.

For an adiabatic process $d Q=0$, therefore eq (1) becomes:

$$
0=d E+P d V
$$

In terms of the system's isometric molar specific heat $\left(C_{\mathrm{v}}\right)$ and the number of moles $(n)$, eq. (2) becomes:

$$
n C_{v} d T+P d V=0
$$

For $n$ moles, the ideal gas law gives: $P=n R T / V$. Substituting into eq. (3):

$$
n C_{v} d T+\frac{n R T d V}{V}=0
$$

Dividing through by temperature $(T)$, eq. (4) then becomes:

$$
n C_{v} d T / T+\frac{n R d V}{V}=0
$$

\section{Challenging (4) AND (5)}

This author has stated that eq. (1) lacks the clarity concerning onto what work is done [3]-[9]. Clarity is obtained by rewriting eq (1) as:

$$
d Q_{\text {in }}=d E_{\text {sys }}+(P d V)_{a t m}
$$

where $d Q_{\text {in }}$ is the energy input, $d E_{\text {sys }}$ is the change to the system's total energy, and ( $P d V)_{\text {atm }}$ is the "lost work" done by an expanding system onto the surrounding atmosphere.

Unfortunately, eq. (3), eq. (4) and eq. (5) are written as if lost work can be expressed in terms of the expanding system's parameters. This author has previously discussed the problematic issues with writing lost work in terms of the expanding system's own parameters. Hence, the clarity of eq. (6) is required [3]-[9].

For an adiabatic process there is no energy in $\left(d Q_{\text {in }}=0\right)$. This leads to:

$$
\left(n C_{v} d T\right)_{s y s}=-(P d V)_{a t m}
$$


If a system undergoes adiabatic expansion, it performs lost work. Therefore, the expanding system's temperature decreases when the system's thermal energy drives the process.

Strangely, eq. (4) and (5) are written in terms of the process being isothermal, which according to eq. (7) is an impossibility. If most adiabatic processes cannot be isothermal, then eq. (4) and (5) are illogical, i.e., describing an adiabatic isothermal expanding system as a general process is void of rationality.

If equations (3) through (5) are irrational, then the rest of what traditionally is accepted concerning adiabatic processes becomes questionable. This includes the following accepted adiabatic process relation [10]:

$$
P d V=-\frac{V d P}{\gamma}
$$

Eq. 8 can be further broken down by applying the adiabatic index: $\gamma=C_{p} / C_{v}$, which leads to:

$$
P d V=-C_{v} V d P / C_{p}
$$

Defining an adiabatic process in terms of eq. (4) through eq. (9) is problematic, if not a completely illogical prospect.

\section{EXothermic Adiabatic Process}

Is there an adiabatic process that can be rationalized? Not if the energy that drives the process comes from the system's internal thermal energy (heat). However, if the energy that drives the process is internal to that expanding system but is not thermal energy, then an adiabatic expanding process is conceivable.

Imagine that an exothermic chemical reaction produces thermal energy $(d U)$ in System 1, as shown in Fig. 1. The energy from this reaction then drives the system's expansion. For such an exothermic reaction in a fully insulated rigid, expandable, experimental apparatus:

$$
d U=-\left[\left(n C_{v} d T\right)_{s y s 1}+(P d V)_{a t m}\right]
$$

In this case, $d T \uparrow, d U \downarrow, d E_{\mathrm{T}}=0$. This assumes that the temperature change is measurable.

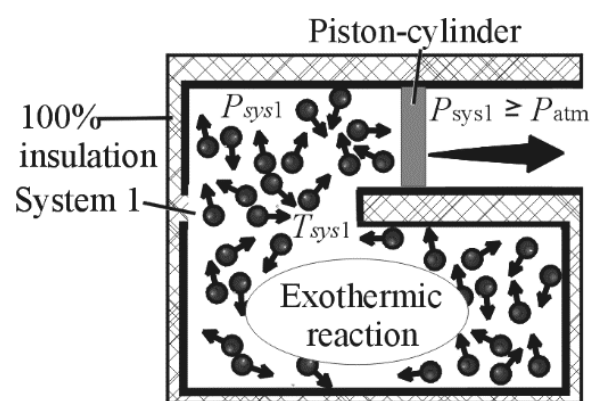

Fig. 1 Shows an exothermic chemical reaction inside of System 1, driving the expansion of gaseous System 1 .

Our relatively new understanding is that the differences between isobaric and isometric heat capacity is due to lost work [3],[4],[7]. This means that eq. (10) can be rewritten in terms of the gas' isobaric molar heat capacity $\left(C_{\mathrm{p}}\right)$ as:

$$
d U=-\left(n C_{p} d T\right)_{s y s 1}
$$

An exothermic reaction becomes a rare case, where one could conceive of a realistic adiabatic expanding process.

\section{FORCED EXPANSION}

Before proceeding it should be stated that the pressuretemperature relationship is now understood to be partially due to the inelastic intermolecular collisions within a system [6][9],[11]-[14]. In other words, as one increases a system's pressure, the amount of thermal energy created by the inelastic intermolecular collisions increases. Therefore, an insulated gaseous system's compression results in its temperature increase. Conversely, in an insulated expanding gaseous system, the natural tendency is for the temperature to decreases as the system's pressure decreases. Such temperature change with pressure change will often be comparatively small, if not infinitesimal.

Furthering our understanding, consider the forced quasistatic expansion of a hermetically sealed piston-cylinder, as illustrated in Fig. 2.

The expanding gas is in an enclosed system, therefore it adheres to the ideal gas law [8],[11]-[13]. The isothermal nature of an uninsulated expanding ideal gas means that thermal energy must be freely-given into the expanding system from the surrounding atmosphere (see $Q_{i n}$ in Fig. 2). Otherwise, as the gas expands, the pressure inside the pistoncylinders decreases and due to the $P-T$ relationship, its temperature should decrease.

Although isothermal, the expanding system shown in Fig. 2 is not an adiabatic process because the expanding force remains external to the system and thermal energy was freelygiven by the atmosphere. Here the surrounding atmosphere acted as an invisible heat bath maintaining the system's isothermal nature.

Is it a reversible process? If one removes the expanding force, then the process will reverse itself and the pistoncylinder will return to its initial state. However, the work required to initially expand the piston-cylinder in Fig. 2 is lost. Therefore, it is an irreversible process although to some it provided the illusion of being reversible.

Specifically, it was lost work, that being work into the surrounding atmosphere, which can be viewed as an irreversible atmospheric energy increase, as defined by, $W_{\text {lost }}=(P d V)_{\text {atm, }}[3]-[9],[11]-[14]$.

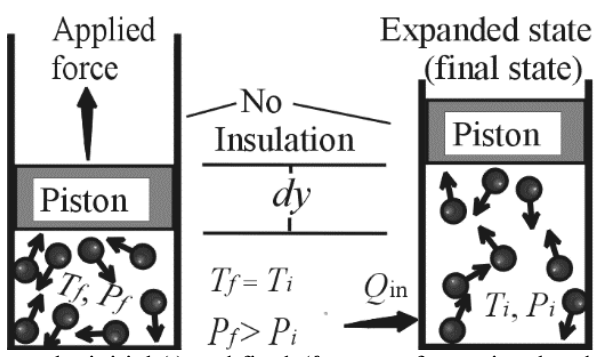

Fig. 2 Shows the initial $(i)$ and final $(f)$ states of an uninsulated gas during forced expansion.

Importantly, when the expanding force is removed and the piston-cylinder contracts, the atmosphere's energy increase as defined by $W_{\text {lost, }}$ is transformed to kinetic energy, which can be viewed as thermal energy (heat) resulting in an 
infinitesimal temperature increase of the atmosphere [3], [6],[7].

\section{FORCED COMPRESSION}

The concept of adiabatic is often applied to the storing of high-pressure gases. Consider that one compresses a gas by an external force, as shown in Fig. 3. If the piston-cylinder is not insulated and the compression is a quasi-static process, thermal energy can escape through the system's walls into the surrounding atmosphere (see $Q_{\text {out }}$ in Fig 3). Accordingly, the compression can appear to be an isothermal process as measured with a thermometer.

In the above isothermal compression, did the gas' energy change? Since it is an enclosed system, the gas can be considered to obey the ideal gas law (so long as it remains sufficiently dilute) [11],[12]. In which case the gas' energy is determined by its temperature. Since its temperature remained constant, the gas' energy did not change.

However, work was done onto the gas, hence the gas' potential to do work $\left[(V d P)_{\text {gas }}\right]$ onto the surrounding atmosphere has increased as defined by:

$$
W_{\text {pot }}=(V d P)_{g a s}
$$

Furthermore, during the gas' compression, some of the surrounding atmospheric gas molecules experienced an increase to their kinetic energy, as they moved down towards Earth's surface. And again, this can be considered as an infinitesimal increase to our atmosphere's temperature.

If the compressing force is removed, then the gas will expand, returning to its original temperature and pressure. In so doing it performs lost work onto the surrounding atmosphere. Is this a reversible process? It may appear to be, but what about the energy that was used to compress the gas? It is now lost work into the atmosphere, therefore isothermal compression is not a reversible process.

Next consider that the piston-cylinder is insulated and the gas within it is compressed. As the gas is compressed, its pressure increases. Therefore, the thermal energy associated inelastic intermolecular collisions increases and the gas' temperature increases.

Accordingly, not only did the gas' potential to do work increase, as defined by eq. (12) but its temperature increased. Therefore, the gas' thermal energy increased. It should be noted that this author's kinetic theory is a superior fit to known empirical findings, when compared to the traditionally accepted kinetic theory [11],[12].

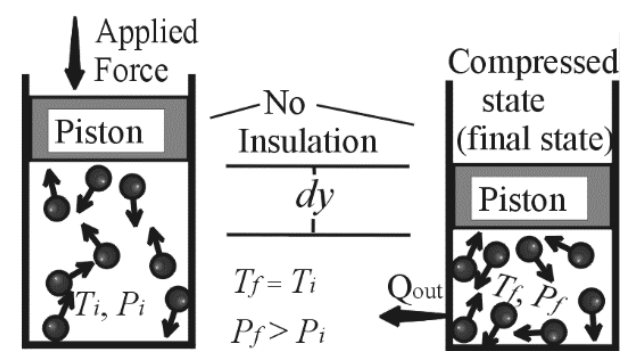

Fig. 3 Shows the initial $(i)$ and final $(f)$ states of an uninsulated gas during compression

Based upon this author's superior fit kinetic theory, an $N$ molecule gas with $n$ " atoms in each molecule, has a total energy [11], [12]:

$$
E_{T} \cong N k T\left(n^{\prime \prime}+1 / 2\right)
$$

Differentiating eq. (13) with respect to temperature gives:

$$
d E_{T}=\left(n^{\prime \prime}+1 / 2\right) N k d T
$$

If the gas is monatomic ( $n "=1)$ then eq. (14) becomes:

$$
d E_{T}=3 N k d T / 2
$$

If the force of compression is removed, the gas will expand. During expansion its temperature will decrease, and it will perform lost work onto the surrounding atmosphere. As was the case for isothermal compression, non-isothermal compression is also an irreversible process because the energy required for the gas' initial compression was lost.

It should be emphasized that the ideal gas law is limited to sufficiently dilute gases in closed systems. Therefore, when compressing such a gas, there will be a point at which the ideal gas law loses it applicability. This occurs when the gaseous intermolecular inelastic collisions dominate over gas-wall molecule inelastic collisions [8],[10],[11].

\section{ISENTROPIC PROCESSES}

An isentropic process is accepted as a process that is both adiabatic and reversible [2],[10]. We have just seen that adiabatic processes are problematic. That being the case, what about isentropic processes $(T d S=0)$ ? Let's investigate

Expanding systems are often described in terms of isothermal entropy change by:

$$
T d S=d E+P d V
$$

Problems with eq. (16) have been discussed by this author [3]-[8],[10-12]. Basically, as is the case for eq. (1), eq. (16) lacks the clarity that eq. (6) possesses. Of course, eq. (16)'s clarity can be improved by writing:

$$
(T d S)_{s y s}=d E_{s y s}+(P d V)_{a t m}
$$

Why write a first law style equation based upon isothermal entropy change $(T d S)$, such as eq. (16)? It starts by realizing that eq. (16) is fundamentally eq. (1) with $T d S$ replacing $d Q$.

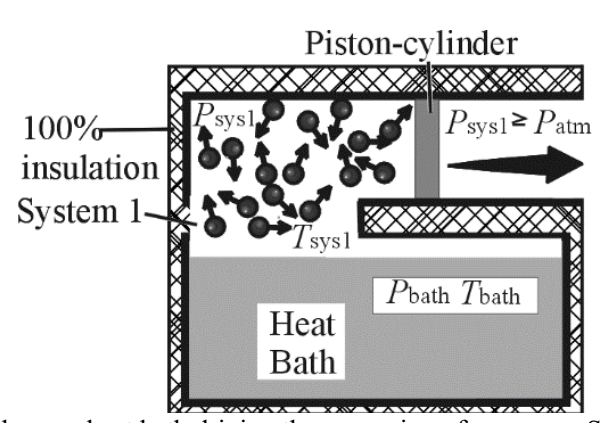

Fig. 4 Shows a heat bath driving the expansion of a gaseous System 1.

Instead of there being a thermal energy input ( $\left.d Q_{\text {in }}\right)$, imagine that an expanding piston-cylinder is connected to a heat bath whose thermal energy drives the expansion of 
gaseous System 1 (see Fig 4). Note that in this case the is no change of state, i.e. no change in bonding potential $(d U=0)$.

The heat transferred from the heat bath into System 1 is $\left[\left(n C_{v} d T\right)_{\text {bath }}\right]$. Accordingly, a first law equation that describes this process is:

$$
\left(n C_{v} d T\right)_{b a t h}=-\left[\left(n C_{v} d T\right)_{s y s 1}+(P d V)_{a t m}\right]
$$

In terms of System 1's isobaric molar heat capacity $\left(C_{p}\right)$, eq. (18) can be rewritten as [see eq. (11)]:

$$
\left(n C_{v} d T\right)_{b a t h}=-\left(n C_{p} d T\right)_{s y s 1}
$$

Consider that the thermal energy associated with the heat bath is orders of magnitude greater than the thermal energy required to increase System 1's temperature plus perform lost work. Thus, there would be an immeasurable decrease in the heat bath's temperature, i.e., $d T_{\text {bath }}=0$. One observing this process might incorrectly assume that in terms of the heat bath, it is an isothermal process.

If one then treats the process as an infinitesimal process, one might also conclude that, $T_{\text {sys }}=T_{\text {bath. }}$. As an isothermal process undergoing infinitesimal change the above suppositions enable one to illogically claim that this process can now be described in terms of isothermal entropy change $(T d S)$, i.e., apply eq. (16).

Consider that Fig. 4 is a real process doing real work. Therefore, the requirement for thermal energy transfer from

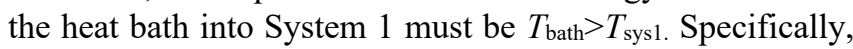
if $T_{\text {bath }}=T_{\text {sys } 1}$, heat is no longer exchanged between the heat bath and System 1 and now it is an adiabatic process as described by eq. (7).

Furthermore, $\left(n C_{v} d T\right)_{\text {bath }}=0$ implies that, $\left(n C_{v} d T\right)_{\text {sys } 1}=0$, therefore there is no lost work, i.e., no actual work could be done. This tells us that the traditional consideration of the process being isothermal is more idealistic than realistic.

As $T_{\text {sys } 1} \rightarrow T_{\text {bath }}$, then the rate of energy exchange must decrease. The rate of thermal energy entering System 1 $(d q / d t)$ can be approximated in terms of some constant $(C)$ :

$$
\frac{d q}{d t}=-\operatorname{CIn}\left(\frac{T_{\text {sys } 1}}{T_{\text {bath }}}\right)
$$

Think of eq. (20) in the following way. Thermal energy flows from both hot to cold, and cold to hot. However, the net direction is always from hot to cold, as measured by a thermometer, i.e. temperature and not entropy determines heat flow's net direct [13]. For two systems of similar materials, this means the net flow is always from high to low thermal energy density.

The requirement for System 1's expansion remains that $P_{\text {sys } 1}>P_{\text {atm, }}$, and, so long as System 1 expands, it performs lost work. Even when connected to a heat bath, there must be a point where both System 1 and the heat bath start to cool down. Furthermore, any flow of thermal energy from the heat bath means that the expansion of System 1 cannot be an adiabatic process.

As $P_{\text {sys } 1} \rightarrow P_{\text {atm, }}$, then the rate of lost work being done decreases. Therefore, the rate of work being done can be approximated in terms of constant $(A)$ by:

$$
\frac{d W}{d t}=-A \operatorname{In}\left(\frac{P_{s y s 1}}{P_{\text {atm }}}\right)
$$

Eq. (20) and eq. (21) demonstrate the requirements of natural logarithmic functionality in thermodynamic modeling. Importantly, these requirements do not involve isothermal entropy change, hence the use of $T d S$ is in dire need of reconsideration.

The reality becomes that expanding systems cannot be isentropic or even adiabatic.

\section{TEMPERATURE-ENTROPY}

One cannot simply berate entropy without obtaining more clarity. Let us consider the simplest of all thermodynamic processes, that being the heating of a solid or liquid. The total energy change of condensed matter in terms of the total energy input $\left(Q_{\text {in }}\right)$, its isometric molar heat capacity $\left(C_{\mathrm{v}}\right)$, and temperature change $(d T)$ is:

$$
d Q_{\text {in }}=n C_{v} d T
$$

Substituting $T d S$ in for $Q_{\text {in }}$ gives:

$$
T d S=n C_{v} d T
$$

This allows for the traditionally accepted:

$$
d S=n C_{v} d T / T
$$

Based upon eq. (24), one obtains an entropy-temperature graph such as that shown on the left side of Fig. 5, i.e., the graph for $S$ vs $T$. The area under the curve becomes the total amount of heat given to the condensed matter. That being $Q_{\text {in }}$.

The amount of heat in $\left(Q_{\text {in }}\right)$ can be viewed another way. Imagine that the rate of heat transfer $(d q / d t)$ was constant. Then for a given duration $(d t)$, one would write:

$$
Q_{\text {in }}=\left(\frac{d q}{d t}\right) d t
$$

Next consider that the rate of thermal energy transfer was not constant. Consider that as the condensed matter becomes hotter, the greater the effort must be in order to add more heat into that substance.
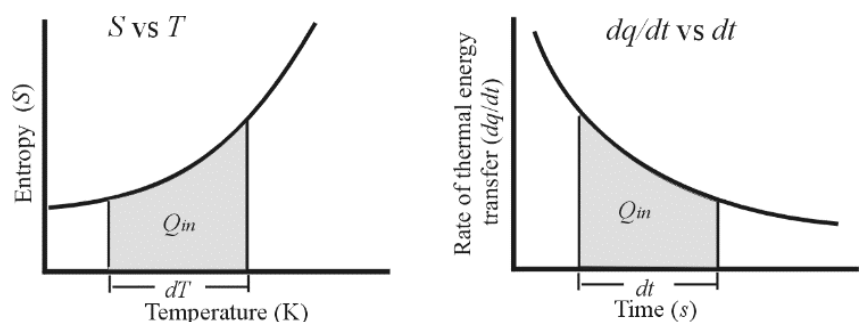

Fig. 5 Shows an $S$ vs $T$ graph for the heating of condensed matter (liquid or solid) on the left side. On the right side is the same heating process but the graph is $d q / d t$ vs $d t$. For both graphs the area under the curve equals the total amount of thermal energy $\left(Q_{\text {in }}\right)$ that is absorbed by the liquid or solid. 
Now the change to the rate that thermal energy transferred into the condensed matter can be described by eq. (20). Substituting eq. (20) into eq. (25) gives:

$$
Q_{\text {in }}=-\operatorname{CIn}\left(\frac{T_{\text {sys } 1}}{T_{\text {bath }}}\right) d t
$$

The total amount of thermal energy into the condensed matter is now shown on the right side of Fig 5, i.e., the graph for $d q / d t$ vs $d t$. The total amount of heat is the area under its graph, which is the same as it is for the much more awkward to visualize entropy-temperature graph, as described by eq. (24). That being $Q_{\text {in }}$.

Could it be that part of the grandiose mistake in thermodynamics was simply not considering the rate of thermal energy decrease, as defined by eq. (20), and that this helps to explain what is witnessed in experiments. In other words, we lost track of the dynamics of time in thermodynamics, replacing it with a poorly-conceived entropy.

Specifically, as the so-called entropy increased, the ability to add more thermal energy decreases. Of course, rather than deal with the quagmire that entropy presents, we could have just thought of the process in terms of rates of energy change.

As extraordinary as that may be, it does seem to be the case.

\section{ENTROPY AND CIRCULAR LOGIC}

Consider that, $\mathrm{A}=\mathrm{B}+\mathrm{C}$ is experimentally proven. Next you decide to set $A=D$, where $D=f(M, N, \ldots R)$, i.e., $D$ is a complex function. Consider that the variables $M, N$...R in function $\mathrm{D}$ are calculated by either directly or indirectly, equating $\mathrm{f}(\mathrm{M}, \mathrm{N}, \ldots \mathrm{R})=\mathrm{B}+\mathrm{C}$.

Can we now say that $\mathrm{D}=\mathrm{B}+\mathrm{C}$ ? It depends. If $\mathrm{A}=\mathrm{D}$ was proven (experimentally?) beyond any doubt, then you can do so with a certain degree of confidence. However, if $\mathrm{A}=\mathrm{D}$ is a mathematical conjecture, then to claim that the complex function proves $\mathrm{B}+\mathrm{C}=\mathrm{D}$ is a circular argument.

It remains this author's assertion that the replacement of the energy input $(d Q)$ in eq. (1) with isothermal entropy change $(T d S)$ resulting in eq. (16), was mathematical conjecture equivalent to the equating of $\mathrm{A}=\mathrm{D}$. This was followed by the creation of a brilliant yet overly-complex mathematics in which entropy was equated to a series of overly-complex functions. In other words, $f(M, N, \ldots R)$ became the foundation of what is known as statistical thermodynamics, which was equated to $T d S$.

Interestingly, this author has previously discussed how Maxwell-Boltzmann distributions may be attributed to what is often witnessed concerning gases in closed systems [13].

Arguably, if clarity was given to eq. (1), as was done with eq. (6), then entropy might not have become the foundation of thermodynamics that it became. Can we now say that traditionally accepted thermodynamics is based upon circular logic? Perhaps we had better say that it is based upon a circular argument that was extremely well-hidden by the enormity of its mathematical complexity.

It has been shown by this author that entropy based thermodynamics is need of a rethink whether one is contemplating work [3]-[9], [11]-[14]or even Helmholtz and Gibbs free energy [9].

\section{ATMOSPHERE AS A HEAT BATH}

The final piece of the puzzle comes from accepting that the atmosphere is a massive heat sink/bath. Therefore, measurable change to its parameters does not occur when considering normal real processes that give or extract thermal energy from it. This explains why our atmosphere is thought of as an isobaric, isothermal, and isometric system. Clearly, if an abundance of real processes occurs, then the atmosphere's parameter change would become measurable, i.e. climate change [14].

Accordingly, although our atmosphere influences almost all systems here on Earth, this fact has gone unnoticed. One can view our atmosphere as the "mother of all heat baths" because:

- $\quad$ it provides freely-given energy to most isothermal expanding systems.

- $\quad$ it absorbs thermal energy from most isothermal systems under compression.

- $\quad$ it provides thermal energy to other heat baths.

- $\quad$ it is the heat bath that all other systems (including experimental heat baths) are compared to [6], [12].

As the mother of all heat baths, our atmosphere's influences upon systems has gone unnoticed far too long.

\section{CONClusion}

Few processes that involve either the compression or the expansion of a gas can be considered as being an adiabatic process. This is due to the fact that expanding systems must do work onto the surrounding atmosphere, that being irreversible work, known as "lost work".

The traditional conceptualization of isentropic processes should be abandoned. Furthermore, the traditional consideration of isothermal entropy is an illogical concept based upon rash and questionable assertions.

Entropy is founded upon a circular argument, that at its core can be replaced with rationalizations concerning changes to the rates of thermal energy change as well as the rate of work.

This means that we must rethink the way we teach and write thermodynamics. A science that is claimed by too many to be a mature science that warrants no challenges.

\section{ACKNOWLEDGMENT}

I would like to thank Lloyd and Gail Mayhew for their assistance in editing this paper.

\section{REFERENCES}

[1] Mayhew, K.W., "Entropy: An ill-conceived mathematical contrivance?", Phys. Essays, 28, 3 (2015), pg 352-357

[2] Reif, F., "Fundamentals of Statistical and Thermal Physics", McGraw-Hill, New York, 1965

[3] Mayhew, K.W., "Resolving Problematic Thermodynamics" Hadronic Journal, Vol 41,4, 2018 pg 257-272

[4] Mayhew, K.W., "New Thermodynamics vs Problematic Traditional", Gen Sci J. Feb., 1, 2019

[5] Mayhew K.W., "Second law and lost work". Phys. Essays, 28, 1 (2015) pp 152-155

[6] Mayhew, K.W., “New Thermodynamics: Temperature, Sun's Insolation, Thermal and Blackbody Radiation", EJERS Vol. 5, 3(2020) pg 264-270 
[7] Mayhew, K.W., "New Thermodynamics: Inefficiency of a PistonCylinder" EJERS Vol 5 Feb 2020 pg 187-191

[8] Mayhew K.W., "New thermodynamics: Illusions of Elastic Collisions in the sciences", EJERS, Vol. 5, 1, (2020), pg 87-90

[9] Mayhew, K.W., "New Thermodynamics: Reversibility and Free Energy", Hadronic Journal, Vol 43, 1, 2020 pg. 51-60

[10] https://en.wikipedia.org/wiki/Adiabatic_process (Apr 2020)

[11] Mayhew, K.W., "A New Perspective for Kinetic Theory and Heat Capacity", Prog. in Phys., Vol. 13 (4) 2017 pg 166-173

[12] Mayhew, K.W., "Kinetic Theory: Flatlining of Polyatomic Gases", Prog. in Phys., Vol. 14 (2) 2018 pg 75-79

[13] Mayhew, K.W., "New Thermodynamics: Understanding Temperature's Limitations" E-J Phys. Vol. 2, 2, (2020) pg. 1-7

[14] Mayhew, K.W., "New Thermodynamics: Global Warming and Man's Activities”, EJERS, Vol. 4, 7, (2019), pg 58-62 